\title{
Lorentz symmetry breaking in the noncommutative Wess-Zumino model: One loop corrections
}

\author{
A.F. Ferrari* and M. Gomes ${ }^{\dagger}$ \\ Instituto de Física, Universidade de São Paulo, Caixa Postal 66318, 05315-970, São Paulo - SP, Brazil \\ H. O. Girotti ${ }^{\ddagger}$ \\ Instituto de Física, Universidade Federal do Rio Grande do Sul, Caixa Postal 15051, 91501-970 - Porto Alegre, RS, Brazil \\ (Received 2 September 2005; published 10 February 2006)

\begin{abstract}
In this paper we deal with the issue of Lorentz symmetry breaking in quantum field theories formulated in a noncommutative space-time. We show that, unlike in some recent analysis of quantum gravity effects, supersymmetry does not protect the theory from the large Lorentz-violating effects arising from the loop corrections. We take advantage of the noncommutative Wess-Zumino model to illustrate this point.
\end{abstract} \\ DOI: 10.1103/PhysRevD.73.047703 \\ PACS numbers: 11.10.Nx, 04.60.Pp, 11.10.Gh, 11.30.Pb
}

It has been common belief that the possible breaking of Lorentz symmetry induced by quantum gravity at the Planck scale is suppressed by enormous ratios at low energies. However, recently, Collins et al. [1] have argued that this is not generally the case. Indeed, using the the Yukawa theory as an example, it was show that quadratically divergent one-loop corrections to the pion self energy translate a breaking of Lorentz symmetry at the Planck scale into an observable violation of Lorentz invariance at low energies. Needless to say, this result is inconsistent with experimental constraints. One way to circumvent this problem is to reestablish the Lorentz symmetry at low energies by adding appropriately adjusted Lagrangian counterterms or, what amounts to the same thing, a Lorentz fine tuning mechanism appears to be required to conciliate theory with experiment.

This Lorentz fine tuning problem is very general and it is present in commutative as well as in noncommutative field theories. With respect to the last ones, the mixing of ultraviolet (UV) and infrared (IR) singularities promoted by the noncommutativity of the spacetime coordinates is known to generate, in nonsupersymmetric models, Lorentz-violating operators which are too large to be consistent with low-energy tests of Lorentz symmetry [2]. In view of these considerations, it was even suggested that (Lorentz-violating) noncommutative field theories are ruled out by the currently available tests of Lorentz invariance [1].

Nevertheless, one might expect to avoid the need of finetuned counterterms to protect Lorentz symmetry at low energies if the theory under consideration is supersymmetric. In the context of commutative field theories, this was shown to be the case in Ref. [3], using the Wess-Zumino model as a prototype. As far as noncommutative models are concerned, supersymmetry is well known to avoid the problem of nonintegrable UV/IR infrared singularities

\footnotetext{
*Electronic address: alysson@ @ma.if.usp.br

${ }^{\dagger}$ Electronic address: mgomes@fma.if.usp.br

"Electronic address: hgirotti@if.ufrgs.br
}

$[4,5]$, thus allowing the construction of a consistent perturbative expansion of these models.

It it natural, therefore, to investigate whether supersymmetry can also prevent the Lorentz fine tuning problem in noncommutative field theories. This paper is dedicated to clarify this question. We use, as arena, the noncommutative Wess-Zumino (WZ) model since, for the time being, it is the only known $(3+1)$-dimensional noncommutative field theory which has been proved to be renormalizable to all orders of perturbation theory $[5,6]$. Furthermore, the fact that all ultraviolet divergences are logarithmic secures that the UV/IR mixing does not give rise to nonintegrable infrared divergences. We focus on the renormalized twopoint vertex function of one of the component fields. The one-loop correction to this vertex function is contributed by planar as well as by nonplanar integrals. The planar integrals are, of course, Lorentz invariant, while the nonplanar ones give, in spite of being ultraviolet finite, a nonvanishing contribution to the quantity $(\xi)$ introduced by Collins et al. [1] as a measure of Lorentz symmetry breaking. Surprisingly, $\xi$ turns out to be fully independent of the intensity of the noncommutativity and, therefore, the calculated value of $\xi$ cannot be put under any experimental constraint. Hence, supersymmetry does not protect the noncommutative WZ model from the Lorentz fine tuning problem.

For the basics of noncommutative quantum field theory we refer the reader to the paper by Minwalla et al. [7] as well as to the review articles [8-11]. We work with the socalled canonical noncommmutavity, where time and position are considered to be self-adjoint operators $\left(q^{\mu}, \mu=\right.$ $0,1,2,3)$ obeying the commutation algebra

$$
\left[q^{\mu}, q^{\nu}\right]=i \Theta^{\mu \nu} .
$$

Here, $\Theta^{\mu \nu}$ is an element of a real numerical antisymmetric matrix $(\|\Theta\|)$, parametrizing the noncommutativity. One can show that it is possible to retain the formulation of the theory in an ordinary (commutative) Minkowski spacetime but deforming the ordinary product between field 
functions into the Grönewold-Moyal $[12,13]$ ( * ) nonlocal product.

We shall be dealing here with noncommutative quantum field theories defined by means of a Lagrangian density in which all ordinary field products are replaced by Grönewold-Moyal ones, and the corresponding Feynman rules are obtained through the usual functional integral methods. Thus, the Feynman propagators remain as in the commutative situation. Only the elementary vertices are modified by the noncommutativity [14]. Observe that in the context of quantum gravity, as studied in $[1,3,15]$, the situation is exactly reversed: the propagators become modified while the vertices remain unchanged.

Already at the tree level, the presence of a constant matrix $(\|\Theta\|)$ has several consequences. Clearly, it breaks Lorentz invariance and, as a consequence, the Lorentz algebra turns out to be deformed by terms proportional to $\Theta^{\mu \nu}$ [16]. Some of the discrete symmetries are also broken, although PCT symmetry is preserved irrespective of the form of $\|\Theta\|$ [17]. The prominent feature is that, at this level, the limit $\Theta^{\mu \nu} \rightarrow 0$ leads us back smoothly to the commutative situation.

Our main concern in this paper is to determine the modifications that the radiative corrections introduce in the picture described above. This can only be done in connection with some specific model. Also, from now on, we assume $\Theta^{0 j}=0$ to evade unitarity and causality problems [18-20].

As we mentioned earlier, in this work we choose the noncommutative WZ model as a prototype of a fully consistent supersymmetric noncommutative theory $[5,6]$. It is defined by the Lagrangian density

$$
\mathcal{L}=\mathcal{L}_{0}+\mathcal{L}_{m}+\mathcal{L}_{g}
$$

where

$$
\begin{aligned}
\mathcal{L}_{0}= & \frac{1}{2} A\left(-\partial^{2}\right) A+\frac{1}{2} B\left(-\partial^{2}\right) B+i \frac{1}{2} \bar{\psi} \phi \psi+\frac{1}{2} F^{2} \\
& +\frac{1}{2} G^{2}, \\
\mathcal{L}_{m}= & m F A+m G B-\frac{m}{2} \bar{\psi} \psi, \\
\mathcal{L}_{g}= & g(F * A * A-F * B * B+G * A * B \\
& \left.+G * B * A-\bar{\psi} * \psi * A-\bar{\psi} * i \gamma_{5} \psi * B\right) .
\end{aligned}
$$

Here, $A$ is a scalar field, $B$ is a pseudo scalar field, $\psi$ is a Majorana spinor field, $F$ and $G$ are, respectively, scalar and pseudoscalar auxiliary fields and $g$ is the coupling constant [21].

We stress that, although Poincaré symmetry is partially broken by the noncommutativity, translations and supersymmetry generators still form a closed undeformed algebra, i.e., supersymmetry is not affected by the presence of the tensor $\Theta^{\mu \nu}[16,22]$. Indeed, one can verify that the action defined by (2) is still invariant under the supersymmetry transformations,

$$
\begin{aligned}
\delta A & =\bar{\alpha} \psi, \\
\delta B & =-i \bar{\alpha} \gamma_{5} \psi, \\
\delta \psi & =-i \not \partial\left(A-i \gamma_{5} B\right) \alpha+\left(F-i \gamma_{5} G\right) \alpha, \\
\delta F & =-i \bar{\alpha} \not \partial \psi, \\
\delta G & =-\bar{\alpha} \gamma_{5} \not \partial \psi,
\end{aligned}
$$

where $\alpha$ is a constant anticommuting Majorana spinor. The proof of invariance of the noncommutative WZ action under (4) is based on the observation that, for any fields $f$ and $g$,

$$
\delta(f * g)=\delta f * g+f * \delta g .
$$

We focus now on the one-loop corrections to the self energy of the field $A$, to be hereafter denoted by $\Gamma(p)$. This object was already computed in Ref. [5] and found to read

$\Gamma(p)=-8 i g^{2} \int \frac{d^{4} k}{(2 \pi)^{4}}(p \cdot k) \cos ^{2}(k \wedge p) \Delta_{F}(k) \Delta_{F}(k+p)$,

where $k \wedge p \equiv \frac{1}{2} k_{\mu} p_{\nu} \Theta^{\mu \nu}$ and $\Delta_{F}(k)$ is the free scalar propagator.

As usual, we split $\Gamma(p)$ into a planar,

$$
\begin{aligned}
\Gamma^{(P)}(p)= & 4 i g^{2} \int \frac{d^{4} k}{(2 \pi)^{4}} \\
& \times \frac{p \cdot k}{\left(k^{2}-m^{2}+i \epsilon\right)\left[(k+p)^{2}-m^{2}+i \epsilon\right]},
\end{aligned}
$$

and a nonplanar contribution,

$$
\begin{aligned}
\Gamma^{(N P)}(p)= & 4 i g^{2} \int \frac{d^{4} k}{(2 \pi)^{4}} \cos (2 p \wedge k) \\
& \times \frac{p \cdot k}{\left(k^{2}-m^{2}+i \epsilon\right)\left[(k+p)^{2}-m^{2}+i \epsilon\right]} .
\end{aligned}
$$

Power counting tell us that $\Gamma^{(P)}(p)$ is UV linearly divergent. However, the would be linearly divergent terms are washed out by symmetric integration. As for the remaining logarithmic UV divergences, one gets rid of them through a renormalization procedure that preserves Lorentz invariance in all stages of the calculation.

We shall focus on the nonplanar contribution, which contains the Lorentz noninvariant factor $\cos (2 p \wedge k)$. The integral in $\Gamma^{(N P)}(p)$ is UV finite and can be explicitly calculated [23]. One obtains

$$
\Gamma^{(N P)}(p)=\frac{g^{2}}{2 \pi^{2}} p^{2} \int_{0}^{1} d x x K_{0}\left(\sqrt{a^{2} p \circ p}\right),
$$

where $K_{0}(z)$ is the modified Bessel function of order zero, $a^{2} \equiv m^{2}-x(1-x) p^{2}$, and

$$
p \circ p=(\vec{p} \cdot \vec{\theta})^{2}-\vec{p}^{2} \vec{\theta}^{2} .
$$

Here, we have introduced the three-vector $\theta^{j}, j=$ $1,2,3$, dual to the antisymmetric tensor $\Theta^{j k}$, namely, 


$$
\Theta_{j k}=\epsilon_{j k l} \theta^{l},
$$

where $\epsilon_{j k l}$ denote the covariant components of the fully antisymmetric Levi-Cività tensor.

According to Eq. (10), the quantity $p \circ p$ may tend to zero because either $\theta=|\vec{\theta}| \rightarrow 0$, or $\vec{p} \rightarrow 0$, or both. If we let $\theta \rightarrow 0$, keeping all components of the four-vector $p^{\mu}$ different from zero, $\Gamma^{(N P)}(p)$ develops a logarithmic singularity. However, if we carry out the same operation in the integrand of Eq. (8) we just recover the commutative counterpart of the two-point vertex function. Hence, the integral in Eq. (8) is not an analytic function of its integrand. This effect is well known to occur in noncommutative field theories and it is at the root of the UV/IR mechanism [7]. Of course, for $p^{\mu} \rightarrow 0$ and $\theta$ arbitrary one gets $\Gamma^{(N P)}(p)=0$. In the case of quantum gravity the role of $\theta$ appears to be played by Planck's cutoff $1 / \Lambda^{2}$. This analogy is based on the fact that these are the dimensional parameters that characterize the scale of Lorentz breaking in each case.

One could also consider the noncommutative theory with an UV cutoff $\Lambda_{U V}$, in which case there would be an additional scale to be taken into consideration. This has been done, for example, in Ref. [2]. In this case, the amount of Lorentz violation found at the low-energy level would depend on the relative strength of the scales of noncommutativity and $\Lambda_{U V}$, as discussed in [2]. For the sake of simplicity, we will not introduce such cutoff, as our conclusions are insensitive to its presence.

We turn now into the problem of quantifying the breaking of Lorentz invariance. It was suggested in Ref. [1] that the appropriate quantity for this purpose is $\xi \equiv$ $\left[\partial^{2} \Gamma^{(N P)}(p) / \partial\left(p^{0}\right)^{2}+\partial^{2} \Gamma^{(N P)}(p) / \partial\left(p^{1}\right)^{2}\right]_{p=0}$. However, for simplifying purposes, it is convenient to adopt the following generalization of this definition,

$$
\xi=\left[\frac{\partial^{2} \Gamma^{(N P)}(p)}{\partial\left(p^{0}\right)^{2}}+\frac{1}{3} \sum_{j=1}^{3} \frac{\partial^{2} \Gamma^{(N P)}(p)}{\partial\left(p^{j}\right)^{2}}\right]_{p=0} .
$$

By combining Eqs. (9) and (12) one finds

$$
\xi=\frac{g^{2}}{3 \pi^{2}} .
$$

Unexpectedly, the breaking of Lorentz invariance does not depend on the intensity $(\theta)$ of the noncommutativity. In particular, $\xi$ remains different from zero even at the limit $\theta \rightarrow 0$.

It is instructive to compare this result with the corresponding one encountered by Collins et al. This essentially implies in replacing the ingredient of noncommutativity by an assumed quantum gravity effect. The model chosen as testing example in Ref. [1] was the Yukawa theory. The interaction Lagrangian is $\mathcal{L}_{g}^{Y}=g \phi \bar{\Psi} \Psi$, where $\phi$ is a real scalar field, while $\Psi$ is a Dirac field. In the standard case, the one-loop contribution to the scalar field self energy
$(\Pi(p))$ would be given by an UV quadratically divergent integral. However, it is assumed that this divergence is regulated by quantum gravity effects at very short distances, and these can be modeled by a cutoff function $f(x)$. This function should verify $f(0)=1$, while vanishing at infinity fast enough to make all Feynman integrals absolutely convergent. With these assumptions in mind, one is allowed to write

$$
\begin{aligned}
\Pi(p)= & -4 i g^{2} \int \frac{d^{4} k}{(2 \pi)^{4}} f\left(\frac{|\vec{k}|}{\Lambda}\right) f\left(\frac{|\vec{p}-\vec{k}|}{\Lambda}\right) \\
& \times \frac{k^{2}-p \cdot k+m^{2}}{\left(k^{2}-m^{2}+i \epsilon\right)\left[(k-p)^{2}-m^{2}+i \epsilon\right]},
\end{aligned}
$$

where $\Lambda$ is a mass of the order of the Planck mass. From this expression, the authors of Ref. [1] obtain

$$
\xi=\frac{g^{2}}{6 \pi^{2}}\left[1+2 \int_{0}^{\infty} d x x f^{\prime}(x)^{2}\right]
$$

Leaving aside of consideration irrelevant numerical factors, the first term in the right hand side of Eq. (15) plays a role analog to that in the right hand side of Eq. (13). Indeed, neither $\theta \rightarrow 0$ nor $\Lambda^{2} \rightarrow \infty$ enables one to recover Lorentz invariance. We see that in both cases the quantum corrections induce large Lorentz breaking contributions at low energies. The source of the problem in the model studied by us is, however, quite distinct from the one considered by Collins et al. since the degree of UV divergence of the starting loop integrals (see Eqs. (8) and (14)) is different.

We recall that, in the commutative situation, supersymmetry act as a custodial symmetry of Lorentz invariance at low energies, thus removing the need for a Lorentz fine tuning mechanism [3]. This happens because supersymmetry secures the cancellation of the leading - quadratic by power counting - contributions to the vertex functions of the quantum theory. The specific model used in Ref. [3] to exemplify this mechanism was, precisely, the WZ model with a Lorentz-violating cut-off function. On the other hand, we have presently used the WZ model to show that, when noncommutativity comes into play, supersymmetry is no longer powerful enough to protect Lorentz invariance at any energy scale. In the noncommutative situation, it is the nonanalyticity induced by the Grönewold-Moyal product which is responsible for the large violation of Lorentz invariance at low energies.

We close this note by recalling some phenomenological studies [2,24,25], where it has been pointed out the existence of difficulties to conciliate canonical noncommutativity with available data on Lorentz symmetry breaking in low-energy experiments. This is due to the generation of Lorentz-violating operators in the noncommutative versions of QED and QCD, like, for instance, the coupling of fermions with a background magnetic field depending on $\|\Theta\|$. However, such theories have problems of their own. In fact, both of them are plagued by quadratic infrared 
divergences [26] arising from the UV/IR mechanism which, as asserted in Ref. [7], invalidate their perturbative expansions. In this connection, the novelty would be to find some inconsistency with Lorentz invariance in a well defined and realistic noncommutative gauge theory. Up to our knowledge, only supersymmetric noncommutative gauge theories have proved to be fully consistent, at least in the one-loop approximation [27]. To provide further back up for this line of argument, we recall that the dangerous Lorentz-violating operators mentioned in the last paragraph of Ref. [25] do not show up in the supersymmetric version of noncommutative QED, if supersymmetry is left unbroken. Of course, it makes no sense to try to conciliate the outcomes from an extremely simple theory, like the noncommutative WZ model, with experimental data.

We can also entertain the hope that alternative approaches to space-time noncommutativity, like the one suggesting the introduction of the tensor $\Theta^{\mu \nu}$ in a
Lorentz covariant way $[28,29]$, may improve the agreement with currently available data. In any case, whether or not we can conciliate space-time noncommutativity with the absence of Lorentz violation in available low-energy experiments is a very interesting question which certainly deserves further study.

A.F. Ferrari is thankful to Professor V. O. Rivelles for discussions. H. O. Girotti and A.F. Ferrari are indebted to F.S. Bemfica for very useful remarks. This work was partially supported by Conselho Nacional de Desenvolvimento Científico e Tecnológico (CNPq) and Fundação de Amparo à Pesquisa do Estado de São Paulo (FAPESP). H.O. Girotti acknowledges support from PRONEX under contract CNPq 66.2002/1998-99. A.F. Ferrari has been supported by FAPESP, project No. 04/ 13314-4.
[1] J. Collins, A. Perez, D. Sudarsky, L. Urrutia, and H. Vucetich, Phys. Rev. Lett. 93, 191301 (2004).

[2] A. Anisimov, T. Banks, M. Dine, and M. Graesser, Phys. Rev. D 65, 085032 (2002).

[3] P. Jain and J. P. Ralston, Phys. Lett. B 621, 213 (2005).

[4] A. Matusis, L. Susskind, and N. Toumbas, J. High Energy Phys. 12 (2000) 002.

[5] H. O. Girotti, M. Gomes, V. O. Rivelles, and A. J. da Silva, Nucl. Phys. B587, 299 (2000).

[6] A. A. Bichl, M. Ertl, A. Gerhold, J. M. Grimstrup, H. Grosse, L. Popp, V. Putz, M. Schweda, and R. Wulkenhaar, Int. J. Mod. Phys. A 19, 4231 (2004).

[7] S. Minwalla, M. van Raamsdonk, and N. Seiberg, J. High Energy Phys. 02 (2000) 020.

[8] M. Douglas and N. A. Nekrasov, Rev. Mod. Phys. 73, 977 (2001).

[9] R. Szabo, Phys. Rep. 378, 207 (2003).

[10] M. Gomes in Proceedings of the XI Jorge André Swieca Summer School, Particles and Fields, edited by G. A. Alves, O.J.P. Éboli, and V. O. Rivelles (World Scientific, Singapore, 2002).

[11] H. O. Girotti, hep-th/0301237.

[12] H. J. Grönewold, Physica 12, 405 (1946).

[13] J. E. Moyal, Proc. Cambridge Philos. Soc. 45, 99 (1949).

[14] See, for instance, Refs. [7-11].

[15] H. Vucetich, gr-qc/0502093.

[16] T. Pengpan and X. Xiong, Phys. Rev. D 63, 085012 (2001).

[17] M. Chaichian, K. Nishijima, and A. Turenu, Phys. Lett. B 568, 146 (2003).
[18] J. Gomis and T. Mehen, Nucl. Phys. B591, 265 (2000).

[19] N. Seiberg, L. Susskind, and N. Toumbas, J. High Energy Phys. 06 (2000) 044.

[20] H. O. Girotti, M. Gomes, A. Yu. Petrov, V. O. Rivelles, and A. J. da Silva, J. High Energy Phys. 05 (2002) 040.

[21] Our Minkowskian metric is $g^{00}=-g^{11}=-g^{22}=$ $-g^{33}=+1$. Furthermore, we use Dirac's representation for the $\gamma$ matrices and $\gamma_{5} \equiv i \gamma^{0} \gamma^{1} \gamma^{2} \gamma^{3}$ implying that $\gamma_{5}^{\dagger}=\gamma_{5}$ and $\gamma_{5}^{2}=1$.

[22] Among several references dealing with this subject we single out C. S. Chu and F. Zamora, J. High Energy Phys. 02 (2000) 022.

[23] I. M. Gradshteyn and I. M. Ryzhik, Tables of Integrals, Series, and Products (Academic Press, Inc., New York, 1980).

[24] C. E. Carlson, C. D. Carone, and R. F. Lebed, Phys. Lett. B 518, 201 (2001).

[25] C. E. Carlson, C. D. Carone, and R. F. Lebed, Phys. Lett. B 549, 337 (2002).

[26] M. Hayakawa, Phys. Lett. B 478, 394 (2000); hep-th/ 9912167.

[27] A. F. Ferrari, H. O. Girotti, M. Gomes, A. Yu. Petrov, A. A. Ribeiro, V. O. Rivelles, and A. J. da Silva, Phys. Rev. D 69 , 025008 (2004); Phys. Rev. D 70, 085012 (2004); A.F. Ferrari, H. O. Girotti, M. Gomes, A. Yu. Petrov, A. A. Ribeiro, and A. J. da Silva, Phys. Lett. B 577, 83 (2003); Phys. Lett. B 601, 88 (2004).

[28] C. E. Carlson, C. D. Carone, and N. Zobin, Phys. Rev. D 66, 075001 (2002).

[29] K. Morita, Prog. Theor. Phys. 108, 1099 (2002). 